\title{
Hyperspectral Image Compression By Using Distributed Source Coding
}

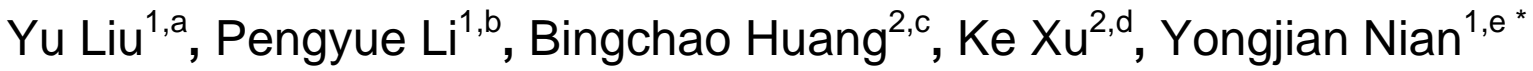 \\ ${ }^{1}$ School of Biomedical Engineering, Third Military Medical University, Chongqing 400038, \\ China \\ ${ }^{2}$ College of Electronic Science and Engineering, National University of Defense Technology, \\ Changsha 410073, China

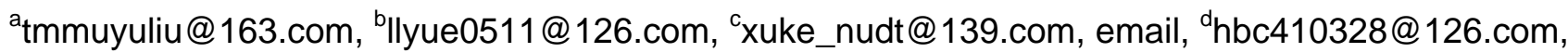 \\ eyjnian@126.com
}

Keywords: Hyperspectral image, lossy compression, distributed source coding, bit-rate allocation. Abstract. This paper presents an efficient compression algorithm based on distributed source coding. The proposed algorithm employs a block-based quantizer followed by distributed lossless coding. First, a bit-rate allocation algorithm is used to assign the rational bit-rate for each block. Subsequently, the multi-linear regression model is employed to construct the side information of each block, and the optimal quantization step size of each block is obtained under the assigned bit-rate while minimizing the distortion. Finally, the quantized block is encoded by distributed lossless compression. Experimental results show that the proposed algorithm provides competitive compression performance, low encoder complexity and error resilience.

\section{Introduction}

Hyperspectral imaging sensors collect data simultaneously in hundreds of narrow and contiguous bands with wavelengths ranging from the visible to the infrared region. At present, hyperspectral imaging has higher spectral and spatial resolution, which leads to a rapid increase in the amount of acquired hyperspectral data. Therefore, developing an efficient compression algorithm for hyperspectral image is an important topic that needs to be solved urgently.

Different from natural images, hyperspectral image have both spectral and spatial correlation. For traditional transform-based lossy compression, spectral decorrelation followed by spatial compression is the most popular manner, with DWT (Discrete Wavelet Transform) and KLT (Karhunen-Loève transform) being two main techniques for spectral decorrelation[1-3]. Although they can provide perfect compression performance, they have high encoder complexity and no error resilience. Distributed source coding (DSC) has received increasing attention from the signal processing field as a new scheme to encode statistically dependent sources, which can provide both low encoder complexity and error resilience, thus satisfying the requirements of onboard compression systems[4,5]. DSC was originally used for lossless compression, based on the Slepian-Wolf theorem[6]. According to this theorem, the lossless compression of the two correlated sources that do not talk each other can be as efficient as if they talk each other, which is true when their compressed outputs are jointly decompressed at a decoder. Wyner A D and Ziv J extended the Slepian-Wolf theory to the field of lossy compression[7]. DSC can be implemented by using binary error-correcting codes or multilevel codes. Pan X Z proposed a low-complexity DSC algorithm based on DCT by using binary error-correcting codes[8]. Tang C M proposed a lossy compression algorithm based on spectral prediction and LDPC (Low Density Parity Check) codes, which can provide low encoder complexity[9]. In [10], Cheung $\mathrm{N} \mathrm{M}$ analyzed the correlation estimation between bit-planes and proposed a model-based estimation algorithm to obtain accurate estimates of bit-plane level correlation subject to complexity constraints. In general, the performance of multilevel codes is better than that of binary error-correcting codes. At present, multilevel codes have been mainly used in lossless compression, while they have scarcely been used in lossy compression. Magli E proposed two DSC-based lossless compression algorithms using multilevel coset codes, referred to as s-DSC and v-DSC[11]. Andrea A proposed three DSC-based lossless compression 
algorithms, providing both low complexity and various degrees of error resilience[12]. Based on s-DSC, an improved distributed lossless compression algorithm is presented in [13], which is also extended to the near lossless compression. In [14], a distributed near lossless compression is proposed; however, it has some shortcomings, firstly, the rate allocation scheme is not perfect; finally, the quantization scheme requires iteration operation for a large number of bands. Based on the algorithm proposed in [14], we propose a new distributed lossy compression algorithm for hyperspectral image, which achieves competitive compression performance, low encoder complexity and error resilience.

\section{The proposed algorithm}

\subsection{Bit-rate allocation}

We first define the weight factor for each block in the spectral orientation as follows

$$
w_{k}=\log _{2} \sigma_{k} / \sum_{l=k}^{L} \log _{2} \sigma_{k}, \quad k=1,2, \ldots, L
$$

where $\sigma_{k}$ is the standard variance of the unquantized errors of the $k$-th block by running the multi-linear regression model. Let $r_{k}$ be the assigned bit-rate of the current block in the $k$-th band and rt be the target bit-rate; the bit-rate allocation is then proposed as follows

$$
r_{k}=\operatorname{round}\left[w_{k}\left(\left(r_{t}-r\right) L-\sum_{l=0}^{k-1}\left(r_{l}-r\right)\right)\right]+r, \quad k=1,2, \ldots, L
$$

where $r_{0}=r=2 \mathrm{bpppb}$. Alternately, when the target bit-rate is below $2 \mathrm{bpppb}$, we first compute the number of blocks that need to be assigned bit-rates as follows

$$
\text { num }=R_{t} L / r
$$

Second, the weight factor of each block is computed according to Eq.1. Finally, the proposed bit-rate allocation algorithm assigns $r$ bpppb to those num blocks having higher weight factors, while the other blocks are skipped without any bit-rate.

\subsection{Block quantization}

Due to the spatially varying nature of hyperspectral image, each band is partitioned into non-overlapping blocks with a size of $N \times N$. Let $x_{k, i, j}$ denote the pixel of the current block in the $i$-th line, $j$-th pixel, and $k$-th band. By employing the multi-linear regression model, the side information of the current pixel $x_{k, i, j}$ is constructed as follows

$$
\bar{x}_{k, i, j}=\sum_{l=1}^{2} \alpha_{l}\left(x_{k-l, i, j}-\mu_{k-l}\right)+\mu_{k}, \quad i, j=1,2, \ldots, N
$$

where $\boldsymbol{\alpha}_{k}$ are the prediction coefficients and $\mu_{k-l}$ is the average value of the co-located block in the $(k-l)$-th band. Once the side information is obtained, the errors can be computed as

$$
e_{k, i, j}=x_{k, i, j}-\bar{x}_{k, i, j}
$$

the LSB number of the block that needs to be transmitted to the decoder is computed as follows

$$
r_{k}=\left\lfloor\log _{2}\left(\max _{i, j=1,2, \ldots, N}\left(\left|e_{k, i, j}\right|\right)\right)\right\rfloor+2
$$

Let $q_{k}$ be the quantization step size of the current block in the k-th band; the quantized version of the current block and its side information are described as follows

$$
y_{k, i, j}=x_{k, i, j} / q_{k}, \bar{y}_{k, i, j}=\bar{x}_{k, i, j} / q_{k}
$$

The error between the current block and its side information is given as

$$
e_{k, i, j}^{\prime}=\operatorname{round}\left(y_{k, i, j}\right)-\bar{y}_{k, i, j}=\operatorname{round}\left(x_{k, i, j} / q_{k}\right)-\bar{x}_{k, i, j} / q_{k}
$$

The error that no more than 0.5 may be introduced as follows 


$$
\delta=\left|\operatorname{round}\left(y_{k, i, j}\right)-y_{k, i, j}\right|
$$

where $\delta$ is ranging from 0 to 0.5 . Thus we can obtain

$$
\operatorname{round}\left(y_{k, i, j}\right)=y_{k, i, j} \pm \delta
$$

It is obviously that the maximum value that error is

$$
\max \left|e_{k, i, j}^{\prime}\right|=\left|y_{k, i, j}-\bar{y}_{k, i, j}+\delta\right|
$$

To ensure the correct reconstruction at the decoder, the following condition must be satisfied

$$
\max \left|e_{k, i, j}^{\prime}\right|<2^{r_{k}-1}, \quad i, j=1,2, \ldots, N
$$

The maximum value of errors should satisfy Eq. 12, i.e.,

$$
\left|y_{k, i, j}-\bar{y}_{k, i, j}+\delta\right|<2^{r_{k}-1}, \quad i, j=1,2, \ldots, N
$$

It is obvious that the following inequation is satisfied

$$
\left|\left(x_{k, i, j}-\bar{x}_{k, i, j}\right) / q_{k}+\delta\right| \leq\left|\left(x_{k, i, j}-\bar{x}_{k, i, j}\right) / q_{k}\right|+\delta
$$

Combining Eq. 13 and Eq. 14, we have

$$
\left|\left(x_{k, i, j}-\bar{x}_{k, i, j}\right) / q_{k}\right|+\delta<2^{r_{k}-1}
$$

As a result, the quantization step size must satisfy

$$
q_{k}>\max _{i, j=1,2, \ldots, N}\left|e_{k, i, j}\right| /\left(2^{r_{k}-1}-\delta\right)
$$

In practice, the optimal quantization step size can be easily determined as

$$
q_{k}=\left\lfloor\max _{i, j=1,2, \ldots, N}\left|e_{k, i, j}\right| /\left(2^{r_{k}-1}-\delta\right)\right\rfloor+1
$$

\section{Experimental results and discussion}

We tested the proposed algorithm on four datasets Cuprite, Jasper Ridge, Lunar Lake and Low Altitude[15]. All the sequences are composed of 224 bands recorded at different wavelengths in the range $380-2500 \mathrm{~nm}$, with a spectral resolution of $10 \mathrm{~nm}$.

\subsection{Compression performance}

The compression results on AVIRIS images by various algorithms are shown in Fig. 1. As seen from Fig. 1, the rate-distortion performance achieved by JPEG2000 Part 1 is the worst because it does not take the spectral correlation into account. JPEG2000 Part 2 (KLT) provides the best rate-distortion performance because KLT can match more closely with the original images. Note that the rate-distortion performance of JPEG2000 Part 2 (DWT) is much lower than that of JPEG2000 Part 2 (KLT) because the basic function of DWT are fixed. As a consequence, a spectral DWT still leaves a significant degree of correlation in the spectral orientation. As for the proposed algorithm, the performance is better than that of JPEG2000 Part 2 (DWT) at high bit-rates. Because the prediction-based technique for spectral decorrelation can adapt to the original data, the performance of spectral decorrelation by using prediction is much better than that by using DWT. Although PCRD-opt can provide excellent rate-distortion performance for JPEG2000 Part 2, the poor performance of spectral decorrelation by using DWT decreases the final rate-distortion performance. However, since the coding technique of the proposed algorithm is so simple, which is much less powerful than that of PCRD-opt, the rate-distortion performance of the proposed algorithm is just slightly higher than that of JPEG2000 Part 2 (DWT). At low bit-rates, the rate-distortion performance of the proposed algorithm is slightly worse than that of JPEG2000 Part 2 (DWT). Because the multi-linear regression model of the proposed algorithm works in sequential manner, it has the shortages of error accumulation at low bit-rates. However, these disadvantages can be completely negligible at high bit-rates. As for JPEG2000 Part 2(DWT) at low bit-rates, the above shortages do not exist, because all of the bands are processed simultaneously. Therefore, the proposed algorithm 
can further improve the quality of the reconstructed images for onboard compression compared with JPEG2000 Part 2 (DWT) at high bit-rates, while provide lower complexity. Note that there is a performance gap between JPEG2000 Part 2 (KLT) and the proposed algorithm. However, this gap is compensated by the capacity of error resilience and the ability to perform parallel encoding.

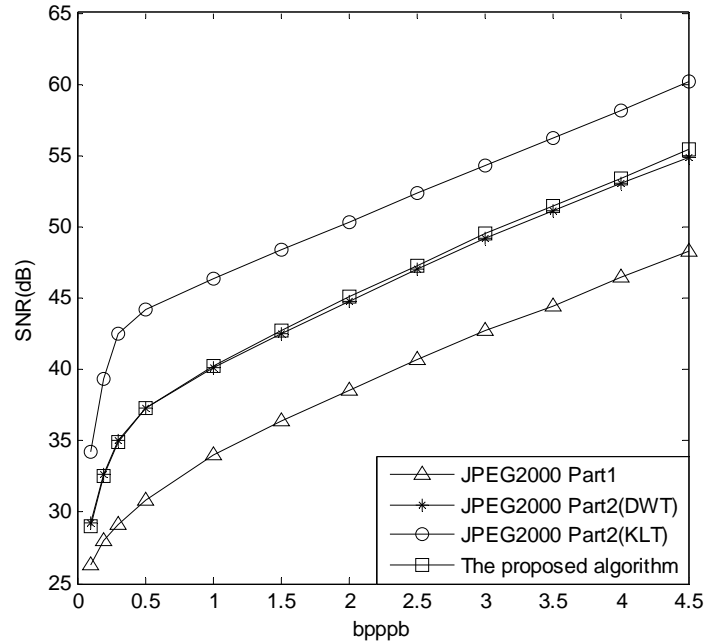

(a) Cuprite

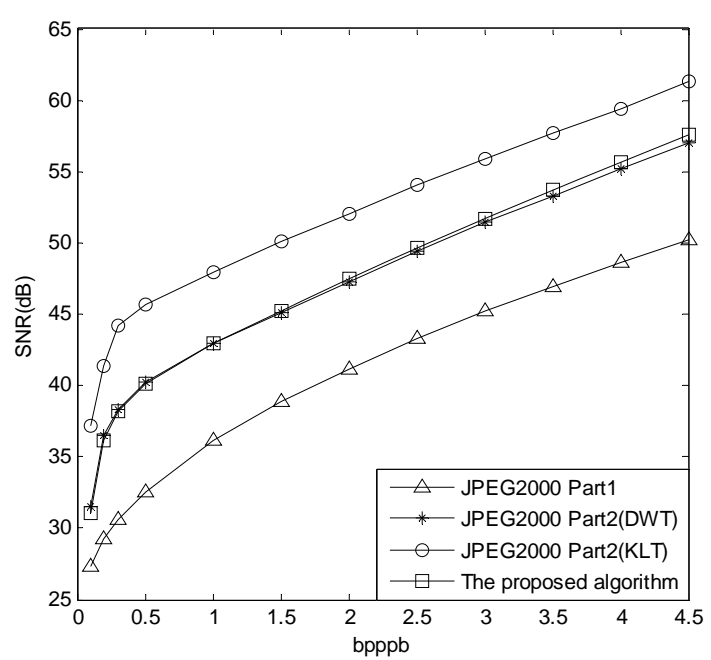

(c) Lunar Lake

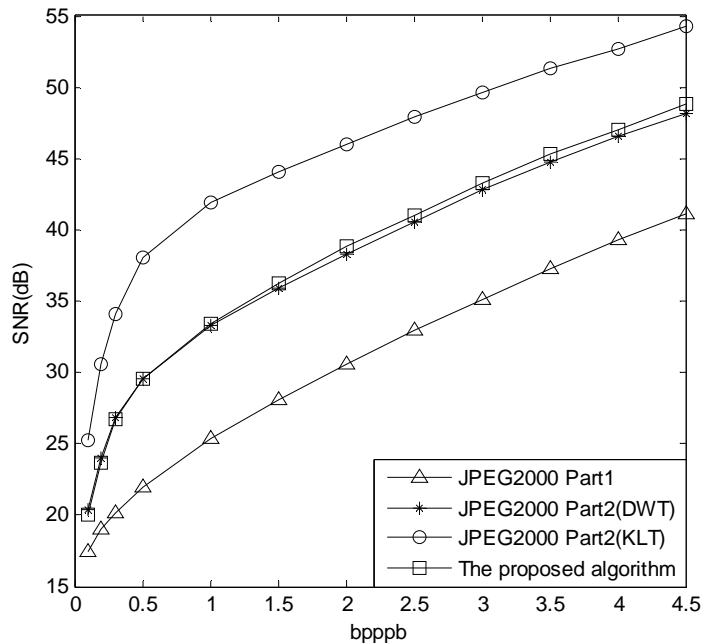

(b) Jasper Ridge

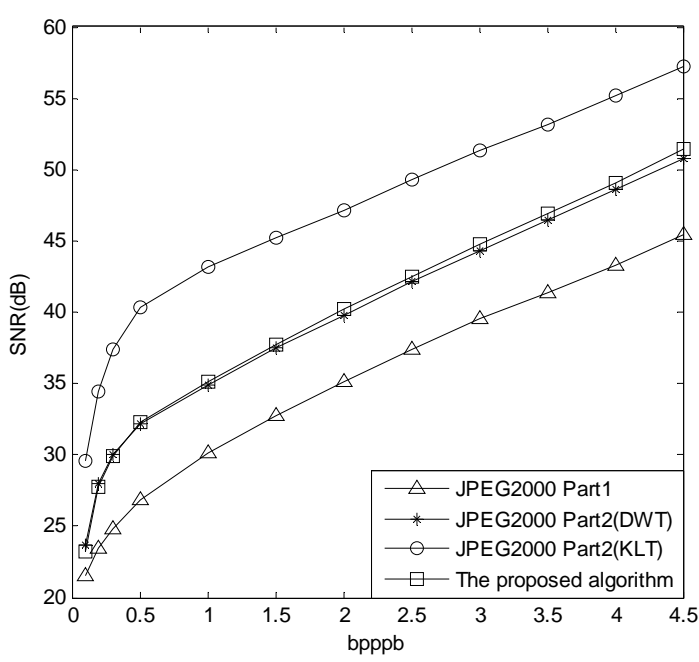

(d) Low Altitude

Fig. 1 The rate-distortion performance

\subsection{Complexity}

In terms of encoder complexity, on average, for the encoding process, the proposed algorithm requires to perform approximately 16 additions and 11 multiplications on each pixel. In terms of memory requirements, JPEG2000 Part 2 usually does not partition bands into blocks because blocks with small sizes are not suitable for spatial transforms, which may decrease the rate-distortion performance. As a result, the required buffer size of JPEG2000 Part 2 is too large because all of the bands need to be transformed simultaneously. For the proposed algorithm, only a buffer of size $N \times N \times L$ is required for each encoding procedure. In summary, the proposed algorithm has similar rate-distortion performance as JPEG2000 Part 2 (DWT), but its encoder complexity is the lowest.

\section{Conclusion}

This paper proposes a lossy compression algorithm based on distributed source coding. Unlike traditional algorithms, the proposed approach is based on the block unit and avoids any transforms, which allows parallelization and yields spatial error containment. The experimental results 
demonstrate that the proposed algorithm has competitive compression performance with the transform-based algorithms. Furthermore, the proposed algorithm provides low encoder complexity and error resilience and allows for easy parallelize implementation, which is suitable for onboard data compression at high throughputs.

\section{Acknowledgments}

This work has been sponsored by the National Natural Science Foundation of China (No. 41201363).

\section{References}

[1] I. Blanes, J. Serra-Sagrista, Pairwise orthogonal transform for spectral image coding, IEEE Transactions on Geoscience and Remote Sensing. 49 (2011) 961-972.

[2] B. Penna, T. Tillo, E. Magli, G. Olmo, Transform coding techniques for lossy hyperspectral data compression, IEEE Transactions on Geoscience and Remote Sensing. 45 (2007) 1408-1421.

[3] K. J. Cheng, J. Dill, Lossless to lossy dual-tree BEZW compression for hyperspectral image, IEEE Transactions on Geoscience and Remote Sensing. 52 (2014) 5765-5770.

[4] S. S. Pradhan, K. Ramchandran, Distributed source coding using syndromes (DISCUS): design and construction, IEEE Transactions on Information Theory. 49 (2003) 626-643.

[5] Z. Xiong, A. D. Liveris, S. Cheng, Distributed source coding for sensor networks, IEEE Signal Processing Magzine. 21 (2004) 80-94.

[6] D. Slepian, J. K. Wolf, Noiseless coding of correlated information sources, IEEE Transactions on Information Theory. 19 (1973) 471-480.

[7] A. D. Wyner, J. Ziv, The bit-rate distortion function for source coding with side information at the decoder, IEEE Transactions on Information Theory. 22 (1976) 1-10.

[8] X. Z. Pan, R. K. Liu, X. Q. Lv, Low-complexity compression method for hyperspectral image based on distributed source coding, IEEE Geoscience and Remote Sensing Letters. 9 (2012) 224-227.

[9] C. M. Tang, N. M. Cheung, A. Ortega, C. S. Raghavendra, Efficient inter-band prediction and wavelet based compression for hyperspectral imagery: a distributed source coding approach, Proceedings of Data Compression Conference, Snowbird, UT, 2005, pp. 437-446.

[10] N. M. Cheung, H. Wang, A. Ortega, Sampling-based correlation estimation for distributed source coding under bit-rate and complexity constraints, IEEE Transactions on Image Processing. 17 (2008) 2122-2137.

[11] E. Magli, M. Barni, A. Abrardo, M. Grangetto, Distributed source coding techniques for lossless compression of hyperspectral image, EURASIP Journal on Advanced Signal Processing. 2007 (2007) 1-13.

[12] A. Abrardo, M. Barni, E. Magli, F. Nencini, Error-resilient and low-complexity onboard lossless compression of hyperspectral image by means of distributed source coding, IEEE Transactions on Geoscience and Remote Sensing. 48 (2010) 1892-1904.

[13] Y. J. Nian, M. He, J. W. Wan, Lossless and near lossless compression of hyperspectral image based on distributed source coding, Journal of Visual Communication and Image Representation. 28 (2015) 113-119.

[14] Y. J. Nian, J. W. Wan, Y. Tang, B. Chen. Near lossless compression of hyperspectral image based on distributed source coding. Science China Information Science. 55 (2012) 2646-2655.

[15] Information on http://aviris.jpl.nasa.gov/data/free_data.html. 